\title{
On the Mechanism of Cross-Dehydrogenative Couplings between $N$ - Aryl Glycinates and Indoles: A Computational Study
}

\author{
Paula Andrade-Sampedro, ${ }^{\mathrm{a}, \mathrm{c}}$ Arkaitz Correa ${ }^{\mathrm{a}, *}$ and Jon M. Matxain ${ }^{\mathrm{b}, \mathrm{c} *}$
}

aUniversity of the Basque Country (UPV/EHU), Department of Organic Chemistry I, Joxe Mari Korta R\&D Center, Avda. Tolosa 72, 20018 Donostia-San Sebastián (Spain)

bPolimero eta Material Aurreratuak: Fisika, Kimika eta Teknologia Saila, Kimika Fakultatea, Euskal Herriko Unibertsitatea (UPV/EHU), Paseo Manuel de Lardizabal 3, 20018 Donostia-San Sebastián (Spain)

'Donostia International Physics Center (DIPC), Paseo Manuel de Lardizabal 4, 20018 Donostia-San Sebastián (Spain)

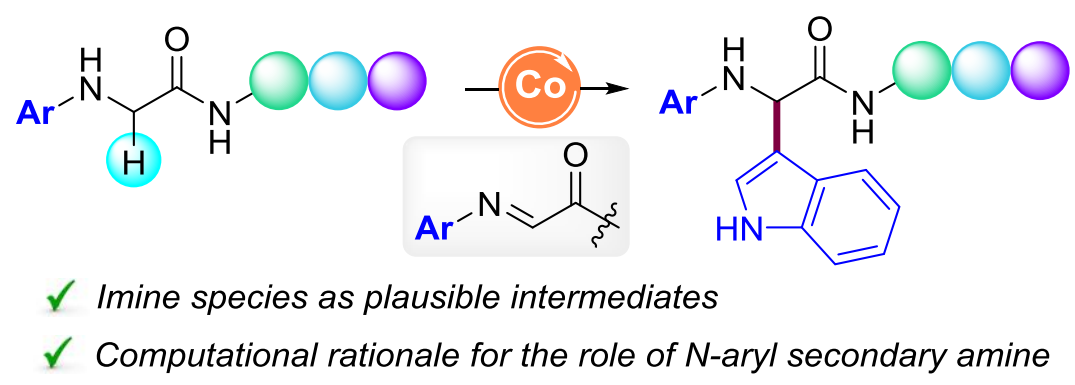

\begin{abstract}
Despite the widespread use of cross-dehydrogenative couplings in modern organic synthesis, mechanistic studies are still rare in the literature and those applied to $\alpha$-amino carbonyl compounds remain virtually unexplored. Herein the mechanism of a Co-catalyzed $\mathrm{CDC}$ of $\mathrm{N}$-aryl glycinates with indoles is described. DFT studies supported the formation of an imine type intermediate as the more plausible transient electrophilic species. Likewise, key information regarding the role of the $\mathrm{N}$-aryl group and free $\mathrm{NH}$ motif within the reaction outcome has been gained, which may set the stage for further developments in this field of expertise.
\end{abstract}

\section{Introduction}

$\mathrm{C}-\mathrm{H}$ functionalization reactions have undoubtedly changed the landscape of modern organic chemistry, thus enabling the development of unprecedented tactics through innovative bond disconnections. ${ }^{1}$ As a result, the modification of otherwise unreactive $\mathrm{C}-\mathrm{H}$ bonds in a predictable and sustainable fashion remains a pressing goal of prime synthetic interest. ${ }^{2}$ In particular, Cross-Dehydrogenative Couplings (CDCs) occurring between two distinct $\mathrm{C}-\mathrm{H}$ reactive sites have recently emerged as atom-economical, yet environmentally friendly techniques for the assembly of a vast array of $\mathrm{C}-\mathrm{C}$ and $\mathrm{C}-$ heteroatom linkages. ${ }^{3}$ They have spawned many advantages including the use of first-row transition metals such as copper, iron or cobalt as efficient catalysts and the production of minimal chemical waste by avoiding the use of pre-functionalized compounds (Scheme 1).

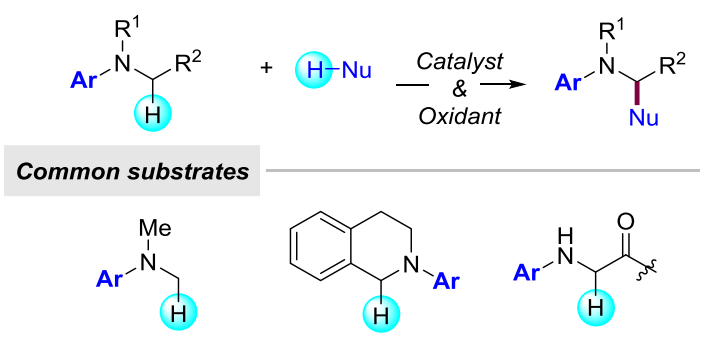

Scheme 1. CDC processes with tertiary and secondary amines

In 2004, Li reported the first oxidative coupling of tertiary amines with terminal alkynes upon copper catalysis. ${ }^{4}$ Since then, CDCs toward the modification of $\alpha-\mathrm{C}-\mathrm{H}$ bonds in adjacent position to nitrogen atoms have received a great deal of attention and has evolved into a powerful platform for forging $\alpha$-substituted amines. ${ }^{5}$ However, despite their widespread use in organic synthesis, mechanistic studies are still rare in the literature and in particular those applied to secondary amines remain virtually unexplored. Accordingly, the understanding of the underlying key elemental steps for the formation of the corresponding electrophilic transient intermediates is of paramount significance and will be pivotal in spurring new applications of CDCs in the years to come at the forefront of organometallic chemistry.

Since the first reports by Miura ${ }^{6}$ and Murahashi ${ }^{7}$ with $\mathrm{Fe}$ - and $\mathrm{Ru}-$ based catalytic systems, respectively, metal-catalyzed oxidative functionalizations of tertiary amines have been posited to proceed via the formation of electrophilic iminium species. Despite the existence of a few comprehensive mechanistic investigations, they rendered divergent mechanistic scenarios and hence the exact mode of activation of the amine derivative still remains a matter of debate. In this light, Klussmann and co-workers ${ }^{8}$ experimentally demonstrated the intermediacy of the iminium ion Int-A derived 
from a direct oxidation by the metal source when using a combination of $\mathrm{CuCl}_{2} / \mathrm{O}_{2}$ as the catalyst/oxidant system and $N$-aryl tetrahydroisoquinolines (THIQs) as substrates (Scheme 2, route a). Further DFT studies supported the formation of the latter species upon a Single Electron Transfer (SET)/ Hydrogen Atom Transfer (HAT) sequence. ${ }^{9}$ Conversely, when using the system $\mathrm{CuBr}$ /tert-butyl hydroperoxide (TBHP), the peroxy species Int-B was shown to be a more plausible intermediate through DFT studies. ${ }^{10}$ On the other hand, studies by Doyle and co-workers ${ }^{11}$ on the use of $\mathrm{N}, \mathrm{N}$-dimethylanilines proposed an initial SET step toward the formation of the ensuing $N$-centered radical cation Int-D which would further undergo a proton transfer (PT)/SET sequence to deliver the corresponding iminium intermediate Int-E (Scheme 2 , route $b$ ). Therefore, it is evident that the conclusions made for a particular system may not be extended to other related ones and the nature of the catalyst, oxidant (oxygen $v s$ organic peroxides) and amine derivative played a determinant role in the reaction pathway.

(a) CDCs with $\mathbf{N}$-aryl THIQs (Klussmann)

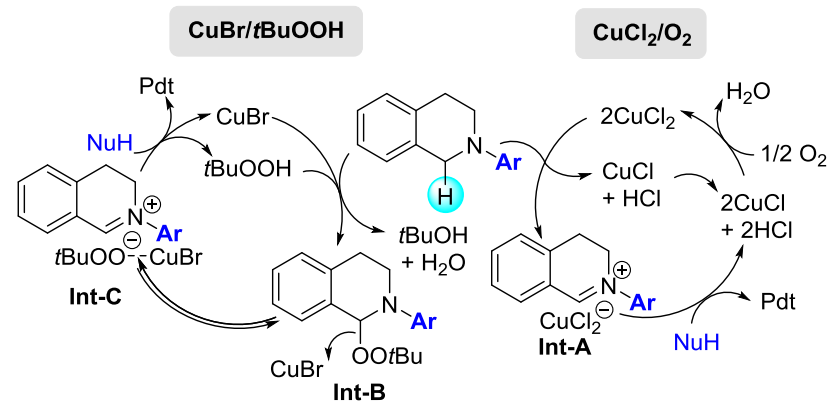

(b) CDCs with N,N-Dimethylanilines (Doyle)

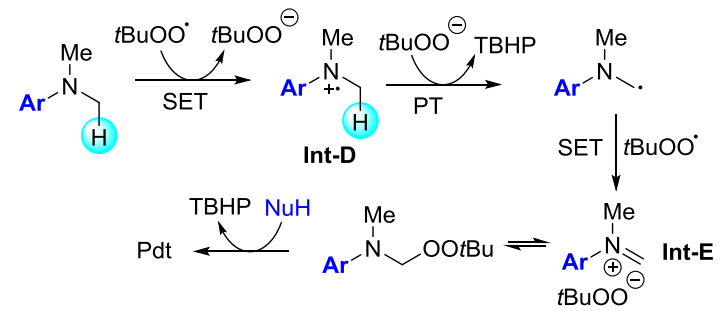

Scheme 2. Proposed mechanisms for CDCs with tertiary amines

Driven by their important biological applications in proteomics and drug discovery, $\alpha$-amino carbonyl compounds have lately emerged as a prominent class of bio-based compounds. ${ }^{12} \mathrm{C}-\mathrm{H}$ functionalization represents a straightforward avenue for the construction of $\alpha$-substituted amino acids and peptides ${ }^{13}$ that are beyond the reach of traditional methods based on solid-phase techniques and carbanion chemistry. Due to the lack of mechanistic studies covering the oxidative coupling of secondary amines upon CDC processes, it is a common practice to propose reaction pathways derived from the extrapolation of the conclusions made with tertiary amine derivatives or indirect experimental evidences. As a result, the formation of an electrophilic $\alpha$-aldimine or $\alpha$-aldiminium intermediate, through the oxidation of the starting $\alpha$-amino carbonyl compound and further reaction with the corresponding nucleophile is generally accepted as a reasonable mechanism (Scheme 3). ${ }^{14}$ However, in-depth investigations are clearly required to gain some insights into the fundamental steps of the process, thereby understanding the unique features as well as the current limitations of the existing CDCs.

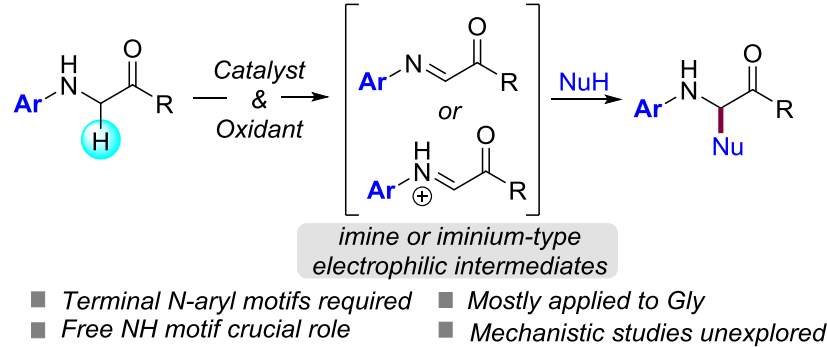

Scheme 3. CDC processes with $N$-aryl Gly compounds

On the one hand, the existing methods mostly allow the modification of terminal $N$-aryl glycine (Gly) units and their corresponding $N$-acyl analogues are rarely utilized. ${ }^{15}$ On the other hand, $N, N$-disubstituted Gly derivatives seem to be unsuitable substrates in these endeavors. In line with our interest in $\mathrm{C}-\mathrm{H}$ functionalization, ${ }^{16}$ we have recently described the efficient $\mathrm{CDC}$ reaction between a variety of $\mathrm{N}$-aryl Gly compounds and indoles featuring the use of an aqueous solution of TBHP and $\mathrm{Co}(\mathrm{acac})_{2}$ as the oxidant and catalyst, respectively. ${ }^{17} \mathrm{On}$ the basis of previous reports, ${ }^{18}$ the intermediacy of an iminium type species was tentatively suggested in our initial report (Scheme 4). The reaction was proposed to start with the $\mathrm{Co}$ (II)-induced decomposition of $t \mathrm{BuOOH},{ }^{19}$ and subsequent HAT would furnish the alkyl radical intermediate Int-F. The latter would be likely converted through a SET event assisted by Co(III) to the electrophilic iminium ion Int-G, which upon reaction with the corresponding indole would eventually deliver the coupling product. The proposed mechanism was merely speculative and further studies were clearly required to rationalize the formation of the electrophilic intermediate, the strong requirement of the terminal $N$-aryl group ${ }^{20}$ and the crucial role that the $\mathrm{NH}$ group seemed to have within the reaction outcome. The latter are indeed common features in a variety of CDCs with amino acids and peptides. ${ }^{14}$ Accordingly, we have performed a computational study of the CDC between $N$-aryl glycinates and indoles in the presence of a $\mathrm{Co}(\mathrm{II}) / \mathrm{t} \mathrm{BuOOH}$ catalytic system and the more important conclusions are disclosed herein. To the best of our knowledge, this study represents the first computational investigation of a metal-catalyzed CDC involving a secondary amine derivative and the obtained results could facilitate the development of new late-stage modification of peptides via CDC techniques. ${ }^{21}$ 


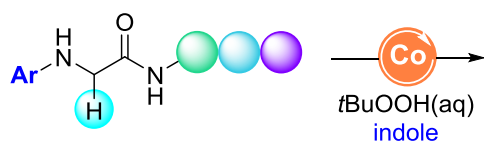

Selected examples

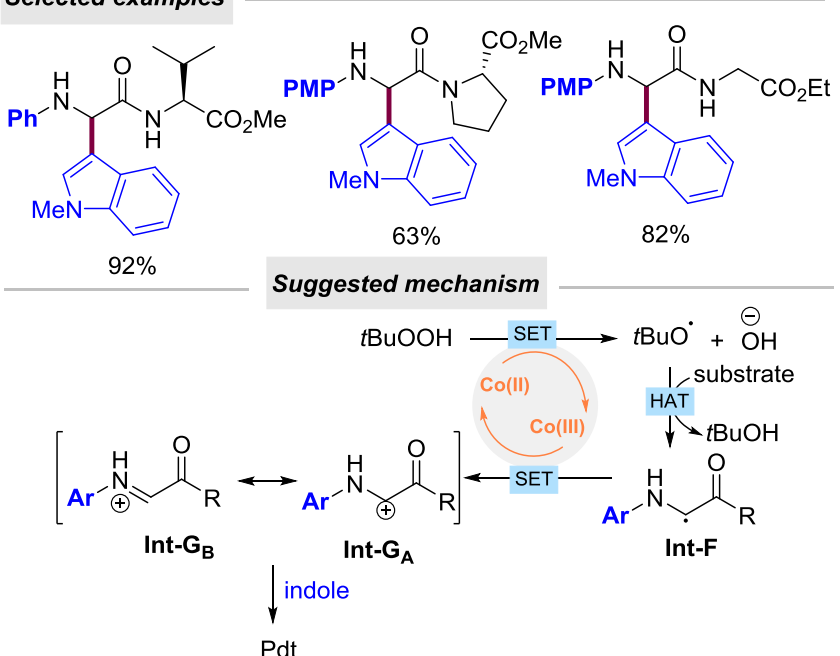

Scheme 4. Co-catalyzed heteroarylation of Gly-containing peptides

\section{Results and Discussion}

It is often assumed that tert-butylhydroperoxide (TBHP) can be easily decomposed under thermal conditions and with the aid of either transition metal salts such $(\mathrm{Cu}, \mathrm{Fe}$ and $\mathrm{Co})$ or iodide salts. ${ }^{22}$ Owing to the redox-active role of the metal, both tert-butoxyl $\left(t \mathrm{BuO}^{\circ}\right)$ and tert-butylperoxyl radicals $\left(t \mathrm{BuOO}^{\circ}\right)$ would likely coexist within our reaction conditions. In principle, any of them could be responsible for the first hydrogen abstraction step (HAT-1) on the Gly derivative $\mathbf{1}$ to produce the carbon-centered radical Int-H (Scheme 5). However, irrespective to the mechanistic nuances, HAT with $t \mathrm{BuO}^{*}$ species have been documented to be faster than the formation of $t \mathrm{BuOO}^{\circ}{ }^{10,23}$ Inspired by the mechanistic studies conducted with tertiary amines ${ }^{8-11}$ different reaction pathways can be envisaged toward the formation of the transient electrophilic iminium or imine species (Scheme 5). First, a direct radical coupling with $t \mathrm{BuOO}^{*}$ would provide Int-I, which has been proposed to exist in equilibrium with the corresponding iminium ion Int-J (route a). Second, oxidation to the carbocation Int-K upon an electron-transfer step (SET-3) (route b). The latter could also be intercepted by the $t \mathrm{BuOOH}$ or its corresponding anion to yield Int-I (route $d$ ). And third, $\mathrm{H}^{\cdot}$ abstraction could furnish a neutral imine intermediate (route c).

Before starting with our computational studies, we conducted some control experiments in order to discard or support the intermediacy of some of the possible transient species depicted above. In this regard, we synthesized the highly reactive imine Int-L-PMP and the $\alpha$-tert-butyldioxyl intermediate Int-I-Ph and submitted to the previously optimized reaction conditions (Scheme 6). The treatment of imine Int-L-PMP with indole afforded the CDC product $\mathbf{3 b}$ in 99\% yield in the absence of both metal and TBHP even at room temperature, thus discarding any role of the latter components within the nucleophilic attack of the indole. On the other hand, peroxyl derivative Int-I-Ph furnished non-functionalized Gly compound $\mathbf{1 a}$ in $41 \%$ yield and just traces of the coupling product $\mathbf{3 a}$ were detected under the standard conditions. As a result, experimental evidences would render unlikely the intermediacy of Int-I and hence route $a$ and route $d$ may not represent a feasible mechanistic scenario. Furthermore, in accordance with literature reports, ${ }^{18 b, 24}$ tertiary amine 4 remained unreactive under the standard conditions and variations on the nature of the metal source or temperature did not result in the formation of the corresponding product.

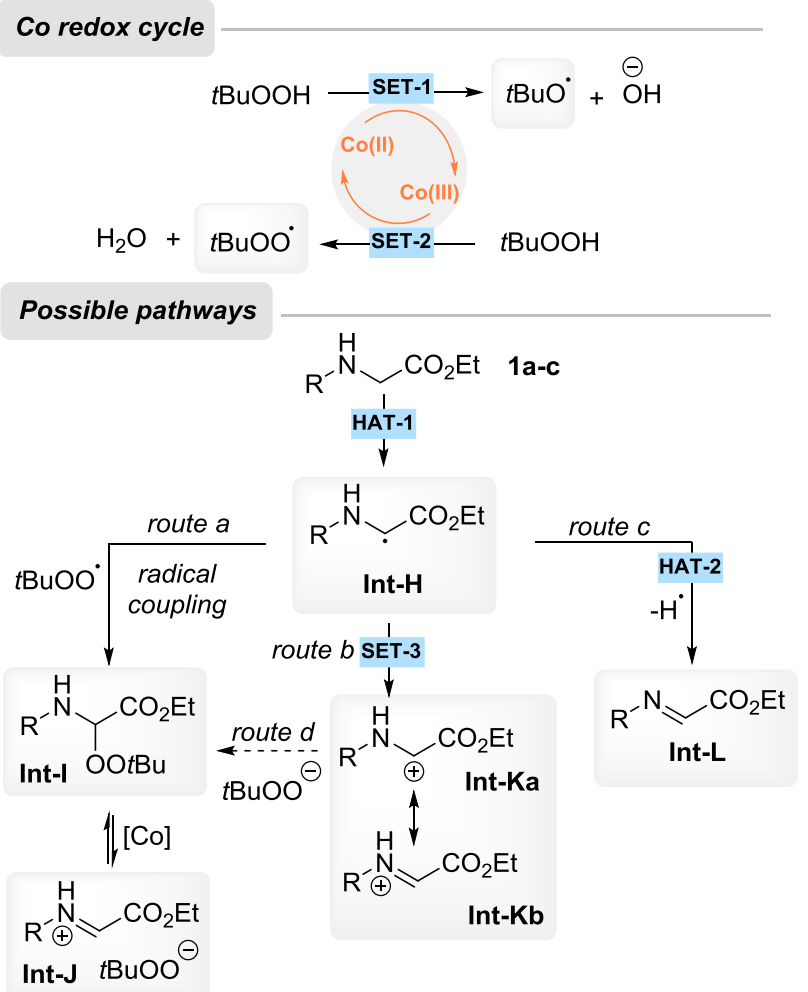

Scheme 5. Co-catalyzed routes toward the electrophilic species

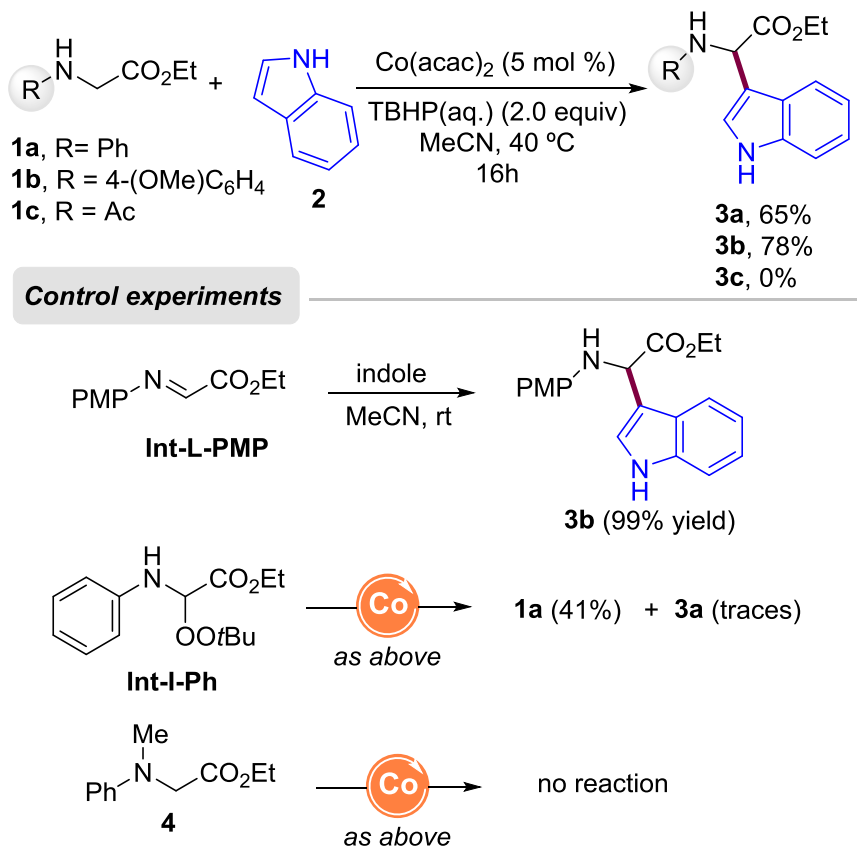

Scheme 6. Control experiments with possible intermediates

Based on the possible reaction mechanisms disclosed in Scheme 5 and the results of the control experiments, different reaction pathways have been considered by means of Density Functional Theory 
(DFT). ${ }^{25}$ The main questions to be answered by these theoretical calculations are the following:

i) Is the imine or the iminium cation pathway preferred?

ii) Which is the actual Co redox cycle?

iii) Why tertiary amines do not react in these CDCs?

iv) Which is the role of the $N$-aryl group?

In order to elucidate all these issues, we focused our calculations on three distinct glycine derivatives (1a-c) (Scheme 6). All the cartesian coordinates of the species mentioned hereafter are given in the Supporting Information, along with the corresponding calculated enthalpies, Gibbs free energies and imaginary harmonic frequencies for Transition States. Notice that along this study, infinitely separated reactants and products are considered along with reactant complexes, transition states, intermediates and products, in order to ensure the continuity of the reaction energy profiles (see Section 3 in SI for further details). ${ }^{25}$ Hence, in order to avoid the unphysical overestimation of entropic effects due to the no inclusion of explicit solvent molecules, ${ }^{26}$ all the energetic discussion will be carried out with enthalpies.

Owing to the good performance of Ph-Gly-OEt (1a) within the Cocatalyzed CDC with indoles, our initial study commenced with the characterization of its full reaction mechanism including a comparative analysis of previously mentioned routes for the CDC with indoles. Before going beyond, however, there are two issues that may be fixed: the most favorable spin states of $\mathrm{Co}$ (II) and $\mathrm{Co}$ (III) species as well the Co redox cycle in our reaction pathway. Regarding the spin states of $\mathrm{Co}(\mathrm{acac})_{2}$ species, doublet and quadruplet planar and tetrahedral species have been considered (Figure 1). According to our calculations, the most stable structure is the tetrahedral quartet spin state, with $2.96 \mathrm{kcal} / \mathrm{mol}$ and $25.83 \mathrm{kcal} / \mathrm{mol}$ difference with respect to the quartet and doublet planar structures, respectively. No tetrahedral doublet structure was found. Hence, we have considered the quartet tetrahedral species as the most stable one for the $\mathrm{Co}(\mathrm{acac})_{2}$ catalyst.
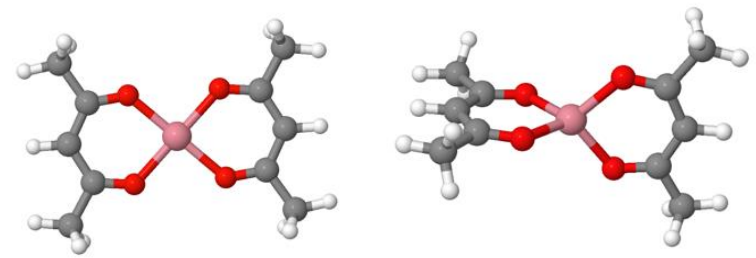

Figure 1. Calculated planar (left) and tetrahedral (right) structures for doublet and quartet spin states of $\mathrm{Co}(\mathrm{acac})_{2}$

As depicted on Scheme 5, the Co(II) catalyst would assist the cleavage of the oxidant, thereby providing the $t \mathrm{BuO}^{\circ}$ radical and the corresponding hydroxylated Co(III) complex upon the SET-1 step. Three possible spin states have been considered for the hydroxylated Co(III) complex: singlet, triplet, and quintet complexes have been calculated and the most stable species is the quintet, being the triplet and singlet states $22.86 \mathrm{kcal} / \mathrm{mol}$ and $29.44 \mathrm{kcal} / \mathrm{mol}$ higher in energy, respectively. This peroxidation process leading to quintet $\mathrm{Co}(\mathrm{acac})_{2} \mathrm{OH}$ complex, commonly referred to as Haber-Weis cycle, has been studied by UV-VIS spectroscopy ${ }^{19 \mathrm{~b}}$ and has an energy penalty of $19.31 \mathrm{kcal} / \mathrm{mol}$. Since the Gly counterpart is not involved, this energy value is common to all Gly compounds regardless of their substitution pattern. In principle, the so-formed
$\mathrm{Co}$ (III)-OH complex may react with $t \mathrm{BuOOH}$, leading to the recovery of the $\mathrm{Co}$ (II) catalyst and the formation of $t \mathrm{BuOO}^{*}$ through the SET-2 step. This process is exothermic by $-11.82 \mathrm{kcal} / \mathrm{mol}$. Accordingly, the Co-redox cycle upon SET-1 and SET-2 would be overall endothermic by $7.49 \mathrm{kcal} / \mathrm{mol}$ and hence the formation of $t \mathrm{BuOO}{ }^{\circ}$ radical species would be unlikely to happen. Consequently, route a could be reasonably discarded. Based on existing precedents and our computational studies, ${ }^{27}$ we concluded that $t \mathrm{BuO}{ }^{\circ}$ species is much more reactive than $t \mathrm{BuOO}^{\circ}$ and that the subsequent HAT event will be more likely to occur with the former radical species.

Having all these considerations in mind, Figure 2 collects the proposed reaction mechanism for Gly derivative 1a. As described before, the first step would be the catalyst oxidation step through SET-1 with an energy penalty of $19.31 \mathrm{kcal} / \mathrm{mol}$, leading to the formation of $t \mathrm{BuO}^{*}$ radical. In this manner, upon initial approach of $t \mathrm{BuO}{ }^{\bullet}$ species to Gly derivative $\mathbf{1 a}$ a reactant complex slightly stabilized with respect to the separated species would be formed (2$\mathrm{RC}$ in Figure 2). The oxygen atom is orientated towards one of the $\alpha$-hydrogen atoms within Gly $\mathbf{1 a}$, and the corresponding hydrogen abstraction has a small barrier of around $5 \mathrm{kcal} / \mathrm{mol}$ respect to the reactant complex, which would lead to the formation of the carboncentered radical Int-H-Ph. Notice that the latter is stabilized through delocalization within the aromatic group and the $\pi$-electron pair of the neighboring nitrogen atom. As a result, intermediate Int$\mathbf{H}-\mathbf{P h}$ is planar. The spin density on the $\alpha-\mathrm{C}$ atom was calculated to be 0.587 and 0.183 in the adjacent nitrogen atom, which underpinned the existing radical delocalization. The formed intermediate is hence stabilized, and located $-26.56 \mathrm{kcal} / \mathrm{mol}$ below the reactant complex of this step and $-10.16 \mathrm{kcal} / \mathrm{mol}$ below the reactants. ${ }^{25}$

Once intermediate Int-H-Ph is formed, two plausible reaction pathways could happen: an oxidation toward iminium ion Int-KPh through SET-3 (Scheme 5, route $b$ ) or a hydrogen abstraction through HAT-2 to deliver imine Int-L-Ph (Scheme 5, route c). The latter are depicted on Figure $2,{ }^{28}$ wherein it is clearly shown that the formation of the iminium cation intermediate Int-K is energetically unfavorable and is located much higher in energy in the potential energy surface. Therefore, the carbon-centered glycine radical Int-H-Ph would be prone to undergo a subsequent HAT-2 step (Scheme 5 , route $c$ ) in order to yield the imine intermediate rather than being oxidized to the ensuing iminium ion Int-K-Ph (Scheme 5 , route $b$ ). In this manner, a Int-H-Ph-Co(III)-OH reactant complex is formed (3RC in Figure 2), which is stabilized by the formation of a hexacoordinated species, where a hydrogen bond is formed between the secondary amine and the $\mathrm{OH}$ of the cobalt complex. 


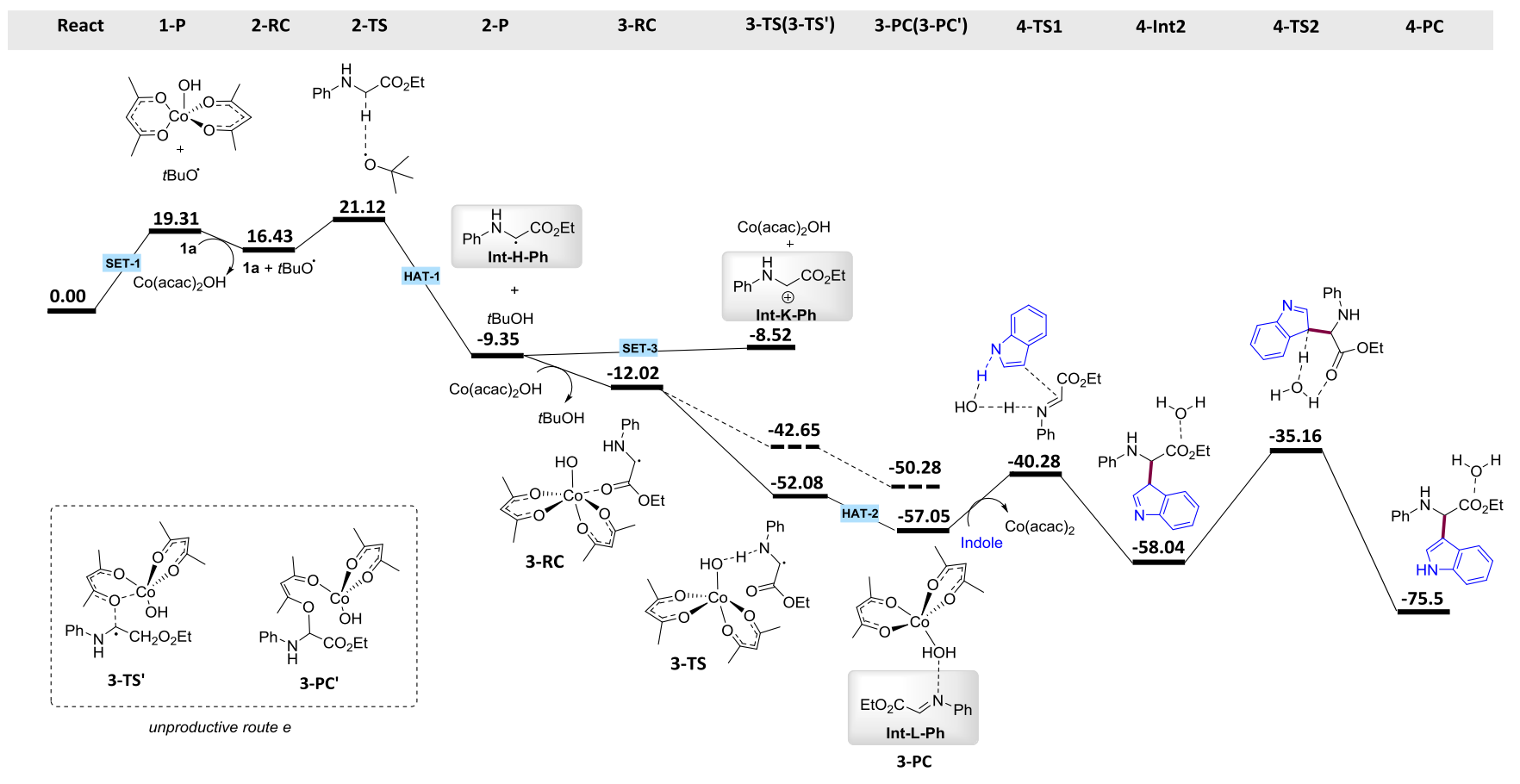

Figure 2. Full reaction mechanism with compound 1a. RC, PC and TS stand for reactant complex, product complex and transition state, respectively, and the number in front accounts for the reaction step; Energy profile $(\Delta \mathrm{H})$ in $\mathrm{kcal} / \mathrm{mol}$.

As shown in Figure 2, this reaction complex $3 \mathrm{RC}$ is ready to undergo a hydrogen abstraction by the hydroxylate ion leading to the recovery of the $\mathrm{Co}(\mathrm{II})$ catalyst, thereby closing the Co-redox cycle, and the formation of a water molecule. This type of reductions of a $\mathrm{Co}(\mathrm{III})-\mathrm{OH}$ complex via hydrogen transfer are known in other catalytic reactions such as decomposition of hydroperoxides. ${ }^{19 b, 29}$ Compared to the initially proposed Co redox cycle where Co(III)$\mathrm{OH}$ could be reduced to $\mathrm{Co}$ (II) with an enthalpy difference of -11 $\mathrm{kcal} / \mathrm{mol}$ upon a SET-2 step (Scheme 5), the formation of the IntL-Ph and recovery of Co(II) catalyst via the HAT-2 step is favored by $-45 \mathrm{kcal} / \mathrm{mol}$, thus $34 \mathrm{kcal} / \mathrm{mol}$ more favorable.

Interestingly, Int-H-Ph-Co(III)-OH reactant complex 3RC could undergo an unexpected and unproductive reaction pathway wherein a new $\mathrm{C}-\mathrm{O}$ bond form is formed between the carbon centered radical and the oxygen atom within one of the acetylacetonate ligands (route $e$ ). The latter is competitive in energy with the HAT-2 process, but the resulting non-reactive adduct lies $7 \mathrm{kcal} / \mathrm{mol}$ above, and the calculated TS barrier is $10 \mathrm{kcal} / \mathrm{mol}$ higher than the HAT2 one. Nevertheless, an intermediate between 3-RC and both TS's is missing. All attempts to locate such intermediate eventually ended on either Int-L-Ph-Co(acac) $)_{2}$ complex, or the alternative mentioned adduct. ${ }^{25}$ Careful analysis underpinned the crucial importance of the electron density of the carbon atom within the radical species Int-H to undergo such an unproductive route and hence the importance of using $N$-aryl groups to minimize the reactivity of the radical intermediate upon effective delocalization (vide infra).

As it will be later explained, the released water molecule may play a key role in the final coupling with the corresponding indole. Importantly, this step is crucial in the reaction mechanism and the hydrogen atom transfer could not occur over tertiary amines, which reasonably explains the experimental evidence that $N$-methyl glycine derivative 4 was not reactive under the standard CDC reaction conditions. This concordance between the theoretical and experimental results reinforces the viability of route $c$ featuring the formation of an imine intermediate.
Once imine intermediate Int-L-Ph is formed, it may undergo further nucleophilic attack by the corresponding indole derivative to furnish the targeted CDC product 3a. Although straightforward at first sight, this attack was found to occur upon a two-step sequence facilitated by the water molecule released along HAT-2. In the first step, indole $\mathbf{2}$ would approach to the electrophilic imine Int-L-Ph, thus forming a reactant complex by hydrogen bonding with the water molecule. Indeed, the water molecule was found to play a dual role and not only enabled the coordination of the electrophile and the nucleophile but also assisted the nucleophilic attack itself. In this respect, imine Int-L-Ph could increase its electrophilicity upon hydrogen bonding with the water molecule, thus allowing the subsequent attack of the indole to forge the new $\mathrm{C}-\mathrm{C}$ bond. In the second step (4-TS2 in Figure 2), rearomatization of the indole motif would deliver the targeted coupling product.

With the aim to clarify the actual role of the phenyl ring in the reaction outcome, the reaction mechanism was also calculated for glycine derivatives $\mathbf{1 b}$ and $\mathbf{1 c}$ bearing a $p$-methoxyphenyl and an acetyl motif at the nitrogen atom, respectively. ${ }^{25}$ The first main difference between the $N$-aryl glycinates $\mathbf{1 a}$ and $\mathbf{1 b}$ and the parent $N$ acetyl derivative 1c is that the carbon-centered radical Int-H is much less stabilized for 1c, which is in accordance with the common trend of utilizing $\mathrm{N}$-aryl compounds in these oxidative functionalization reactions. Notice that the products of the formation of the radicals lie around $6-7 \mathrm{kcal} / \mathrm{mol}$ higher in energy in comparison to the $\mathrm{N}$-aryl substituted radicals. ${ }^{25}$ In particular, the stabilization of the aromatic radicals Int-H-Ph and Int-H-PMP is due to the effective delocalization of the radical. Spin densities for the $\alpha$-carbon atom show values of $0.587,0.563$ and 0.708 for Int-H-Ph, Int-HPMP and Int-H-Ac, respectively. Based on these values, it can be concluded that the radical is more delocalized in $N$-aryl derivatives. Recall that the more delocalized the radical is, the higher its stability. This stabilization effect is also observed in the formation of the Int-H-Co(III)-OH complex and the corresponding imine intermediate Int-L, which are more stable for $N$-aryl derivatives by roughly $15 \mathrm{kcal} / \mathrm{mol}$. The higher stabilization of the imine derivatives IntL-Ph and Int-L-PMP in comparison with Int-L-Ac shows that the 
hydrogen atom transfer occurring in this step is more difficult for 1c than for the parent aromatic compounds $\mathbf{1 a}$ and $\mathbf{1 b}$. In fact, owing to the higher electron density of the carbon radical of Int-H-Ac derived from the $\mathrm{N}$-acetyl glycine derivative 1c, in this case complex 3RC would preferentially evolve into the formation of a new adduct through route $e$ instead of the expected HAT-2 with the concomitant reduction of the Co(III) catalyst (Scheme 7).

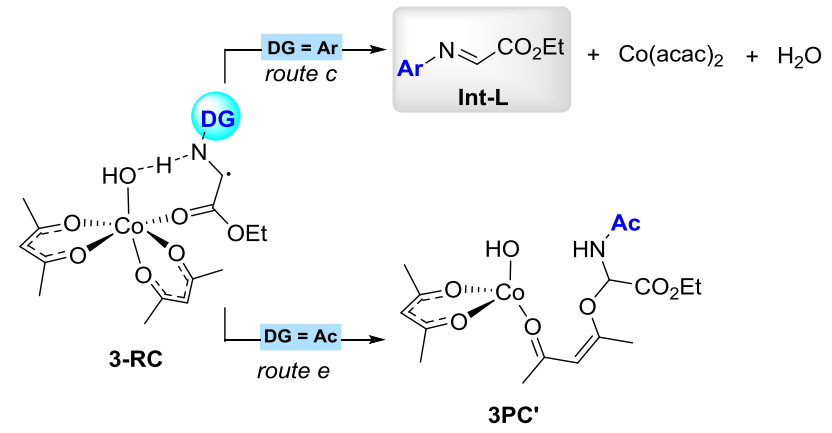

Scheme 7. Distinct reactivity of $N$-aryl and $N$-acyl derivatives

Despite the similar energy values toward the formation of Int-H, the presence of an $\mathrm{N}$-acetyl group clearly favors the unproductive route e. Conversely, in the case of the $N$-aryl compounds, Int-L$\mathbf{P h}$ and Int-L-PMP are the preferred products through route $c$ (Figure 3). Consequently, the formation of Int-L-Ac would be frustrated due to the high spin density concentration in the $\alpha$-carbon. According to these results, the experimental evidence of the higher reactivity of aromatic amine derivatives versus the lack of reactivity of the acyl analogues may be reasonably explained. In fact, a wide variety of extra experiments were performed with $N$-acyl glycine derivative 1c (Table S1, Supporting Information), but the target CDC product $3 \mathbf{c}$ was never detected, which reinforced the hypothesis that an aromatic moiety within the $N$-terminal position is required for the process to occur. On balance then, we could conclude that the formation of the imine species Int-L upon route $c$ is the preferred pathway to obtain the CDC product, which is favored by the presence of aryl groups within the terminal nitrogen atom.

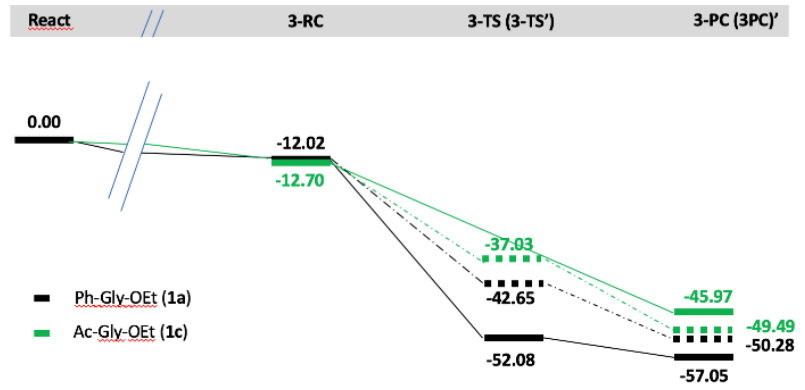

Figure 3. Comparison of the reaction energetics (in $\mathrm{kcal} / \mathrm{mol}$ ) for $1 \mathbf{a}$ and $\mathbf{1 c}$ within route $c$ (straight line) and route $e$ (dashed line)

Based on the DFT studies disclosed above, we revisited the originally proposed reaction mechanism featuring the intermediacy of iminium species, ${ }^{14,17}$ and the most feasible reaction pathway involving a neutral imine intermediate is disclosed in Scheme 8. The reaction was proposed to start with the $\mathrm{Co}$ (II)-induced cleavage of $t \mathrm{BuOOH}$ to produce the active $t \mathrm{BuO}^{*}$ species, which would further deliver a carbon-centered alkyl radical upon a HAT event. The lat- ter would undergo a more energetically favored hydrogen abstraction by the Co(III) species, thus closing the catalyst cycle and delivering the electrophilic imine intermediate. Eventually, the nucleophilic attack of the indole would furnish the target coupling product, which seems to be favored by the water molecule released along the HAT-2.

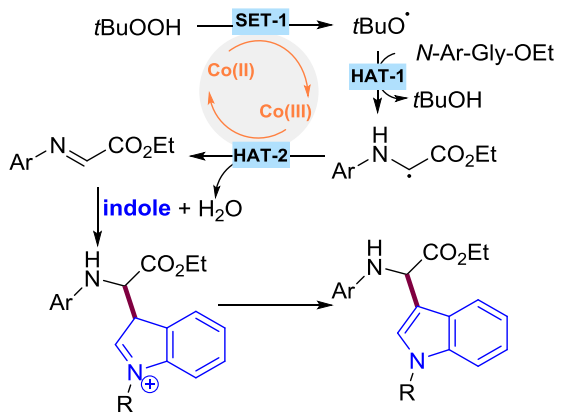

Scheme 8. Proposed revised mechanism for the Co-catalyzed heteroarylation of $\mathrm{N}$-aryl glycinates

\section{Conclusions}

In summary, we have investigated the mechanism of Co-catalyzed $\mathrm{CDC}$ of $N$-substituted glycinates with indoles by DFT studies. Although merely speculative, existing reports commonly proposed the intermediacy of both imine and iminium-type species in these endeavors; however, the nature of the actual electrophilic intermediate remained unclear. Our computational studies supported the formation of a neutral imine species as the more favorable pathway in the Co-catalyzed heteroarylation of glycine-containing compounds. Likewise, this route seemed to be favored when using $\mathrm{N}$ aryl substituted derivatives and secondary amines as substrates due to the strong delocalization of the $\alpha$-carbon centered radical intermediate. These computational studies are in full agreement with the common practice of performing CDC reactions with $\mathrm{N}$-aryl glycine compounds and the rationale described herein could lay the foundation for further developments in this field of expertise.

\section{Experimental Section}

Control Experiments with Preformed Intermediate Int-LPMP: A reaction tube containing a stirring bar was charged with imine Int-L-PMP $(0.25 \mathrm{mmol}, 103 \mathrm{mg})$ and indole $(0.25 \mathrm{mmol}$, $29.3 \mathrm{mg}$ ). The reaction tube was then evacuated and back-filled with dry argon (this sequence was repeated up to three times). Then, MeCN ( $1 \mathrm{~mL})$ was added under argon atmosphere and the reaction was stirred at room temperature for 24 hours. The mixture was then concentrated under reduced pressure and purified by flash chromatography (hexanes/EtOAc 9/1) to deliver $80 \mathrm{mg}$ (99\% yield) of $\mathbf{3 b}$ as a brownish oil. The spectroscopic data corresponded to those previously reported in the literature. ${ }^{17}$

Control Experiments with Preformed Intermediate Int-I-Ph: A reaction tube containing a stirring bar was charged with Int-I-Ph ${ }^{17}$ $(0.50 \mathrm{mmol}, 134 \mathrm{mg}), \mathrm{Co}(\mathrm{acac})_{2} \cdot \mathrm{H}_{2} \mathrm{O}(5 \mathrm{~mol} \%)$ and indole $(1.00$ $\mathrm{mmol}, 117 \mathrm{mg})$. The reaction tube was then evacuated and backfilled with dry argon (this sequence was repeated up to three times). Then, dry MeCN (1 mL) and an aqueous solution of TBHP (70 wt. $\%$ in $\mathrm{H}_{2} \mathrm{O}$ ) (2.00 equiv.) were added under argon atmosphere. The reaction tube was next warmed up to $40^{\circ} \mathrm{C}$ and stirred for 16 hours. The mixture was then allowed to warm to room temperature, concentrated under reduced pressure and purified by flash chromatography (Hexane/EtOAc, 8/2) to deliver $37 \mathrm{mg}$ (41\% yield) of product $1 \mathbf{a}$ as a white solid and just traces of coupling product $\mathbf{3 a}$. 


\section{ASSOCIATED CONTENT}

\section{Supporting Information}

The Supporting Information is available free of charge on the ACS Publications website at http://pubs.acs.org.

Energetics and Cartesian Coordinates of the Optimized Structures (PDF).

\section{AUTHOR INFORMATION}

\section{Corresponding Author}

*arkaitz.correa@ehu.eus

*jonmattin.matxain@ehu.eus

\section{ORCID}

Paula Andrade-Sampedro: 0000-0002-6643-1052

Arkaitz Correa: 0000-0002-9004-3842

Jon M. Matxain: 0000-0002-6342-0649

\section{Notes}

The authors declare no competing financial interests.

\section{ACKNOWLEDGMENT}

We are grateful to Ministerio de Ciencia e Innovación (RTI2018093721-B-I00, MCI/AEI/FEDER, UE) and Basque Government (IT1033-16 and IT1254-19) for financial support. We thank for technical and human support provided by SGIker of UPV/EHU and European funding (ERDF and ESF).

\section{REFERENCES}

1. For selected reviews, see: (a) Rej, S.; Ano, Y.; Chatani, N. Bidentate Directing Groups: An Efficient Tool in $\mathrm{C}-\mathrm{H}$ Bond Functionalization Chemistry for the Expedient Construction of C-C Bonds. Chem. Rev. 2020, 120, 1788. (b) Karimov, R. R.; Hartwig, J. F. Transition-Metal-Catalyzed Selective Functionalization of $\mathrm{C}\left(\mathrm{sp}^{3}\right)-\mathrm{H}$ Bonds in Natural Products. Angew. Chem. Int. Ed. 2018, 57, 4234. (c) Chu, J. C. K.; Rovis, T. Complementary Strategies for Directed $\mathrm{C}\left(\mathrm{sp}^{3}\right)-\mathrm{H}$ Functionalization: A Comparison of Transition-Metal-Catalyzed Activation, Hydrogen Atom Transfer, and Carbene/Nitrene Transfer. Angew. Chem. Int. Ed. 2018, 57, 62. (d) He, J.; Wasa, M.; Chan, K. S. L.; Shao, Q.; Yu, J.-Q. Palladium-Catalyzed Transformations of Alkyl C-H Bonds. Chem. Rev. 2017, 117, 8754. (e) Hartwig, J. F.; Larsen, M. A. Undirected, Homogeneous C-H Bond Functionalization: Challenges and Opportunities. ACS Centr. Sci. 2016, 2, 281. (f) Wencel-Delord, J.; Glorius, F. C$\mathrm{H}$ Bond Activation Enables the Rapid Construction and LateStage Diversification of Functional Molecules. Nat. Chem. 2013, 5, 369. (g) Newhouse, T.; Baran, P. S. If C-H Bonds Could Talk: Selective C-H Bond Oxidation. Angew. Chem. Int. Ed. 2011, 50, 3362.

2. (a) Hayler, J. D.: Leahy, D. K.; Simmons, E. M. A Pharmaceutical Industry Perspective on Sustainable Metal Catalysis. Organometallics 2019, 38, 36. (b) Gandeepan, P.; Müller, T.; Zell, D.; Cera, G.; Warratz, S.; Ackermann, L. 3d Transition Metals for C-H Activation. Chem. Rev. 2019, 119, 2192. (c) Tzouras, N. V.; Stamatopoulos, I. K.; Papastavrou, A. T.; Liori, A. A.; Vougioukalakis, G. C. Sustainable Metal Catalysis in CH Activation. Coord. Chem. Rev. 2017, 343, 25.
3. For selected reviews, see: (a) Huang, C.-Y.; Kang, H.; Li, J.; Li, C.-J. En Route to Intermolecular Cross-Dehydrogenative Coupling Reactions. J. Org. Chem. 2019, 84, 12705. (b) Parvatkar, P. T.; Manetsch, R.; Banik, B. K. Metal-Free Cross-Dehydrogenative Coupling (CDC): Molecular Iodine as a Versatile Catalyst/Reagent for CDC Reactions. Chem. Asian J. 2019, 14, 6. (c) Yi, H.; Zhang, G.; Wang, H.; Huang, Z.; Wang, J.; Singh, A. K.; Lei, A. Recent Advances in Radical C-H Activation/Radical Cross-Coupling. Chem. Rev. 2017, 117, 9016. (d) Lv, L.; Li, Z. Fe-Catalyzed Cross-Dehydrogenative Coupling Reactions. Top. Curr. Chem. 2016, 374, 38. (e) Girard, A. A.; Knauber, T.; Li, C.-J. The Cross-Dehydrogenative Coupling of C-H Bonds: A Versatile Strategy for $\mathrm{C}-\mathrm{C}$ Bond Formations. Angew. Chem. Int. Ed. 2014, 53, 74.

4. Li, Z.; Li, C.-J. CuBr-Catalyzed Efficient Alkynylation of $\mathrm{sp}^{3}$ $\mathrm{C}-\mathrm{H}$ Bonds Adjacent to a Nitrogen Atom. J. Am. Chem. Soc. 2004, 126, 11810.

5. Dai, X.-J.; Li, C.-J. In Comprehensive Organic Synthesis; (Ed.: P. Knochel, G. A. Molander), Elsevier, Amsterdam, 2014, 242.

6. Murata, S.; Miura, M.; Nomura, M. Iron-Catalysed Oxidation of $N, N$-Dimethylaniline with Molecular Oxygen. J. Chem. Soc. Chem. Commun. 1989, 116.

7. Murahashi, S.-L.; Komiya, N.; Terai, H.; Nakae, T. Aerobic Ruthenium-Catalyzed Oxidative Cyanation of Tertiary Amines with Sodium Cyanide. J. Am. Chem. Soc. 2003, 125, 15312.

8. (a) Boess, E.; Schmitz, C.; Klussmann, M. A Comparative Mechanistic Study of Cu-Catalyzed Oxidative Coupling Reactions with $N$-Phenyltetrahydroisoquinoline. J. Am. Chem. Soc. 2012, 134, 5317. (b) Boess, E.; Sureshkumar, D.; Sud, A.; Wirtz, C.; Farès, C.; Klussmann, M. Mechanistic Studies on a $\mathrm{Cu}-\mathrm{Catalyzed}$ Aerobic Oxidative Coupling Reaction with $\mathrm{N}$ Phenyl Tetrahydroisoquinoline: Structure of Intermediates and the Role of Methanol As a Solvent. J. Am. Chem. Soc. 2011, 133,8106 .

9. (a) Morgante, P.; Dughera, S.; Ghigo, G. Aerobic $\mathrm{CuCl}_{2}$-Catalyzed Dehydrogenative Cross-Coupling of Tertiary Amines. A Combined Computational and Experimental Study. J. Phys. Chem. A 2019, 123, 2796. (b) Bartling, H.; Eisenhofer, A.; König, B.; Gschwind, R. M. The Photocatalyzed Aza-Henry Reaction of N-Aryltetrahydroisoquinolines: Comprehensive Mechanism, $\mathrm{H}^{\circ}$ - versus $\mathrm{H}^{+}$-Abstraction, and Background Reactions. J. Am. Chem. Soc. 2016, 138, 11860. (c) Cheng, G.-J.; Song, L.-J.; Yang, Y.-F.; Zhang, X.; Wiest, O.; Wu, Y.-D. Computational Studies on the Mechanism of the Copper-Catalyzed $\mathrm{sp}^{3}-\mathrm{C}-\mathrm{H}$ Cross-Dehydrogenative Coupling Reaction. ChemPlusChem 2013, 78, 943.

10. Boess, E.; Wolf, L. M.; Malakar, S.; Salamone, M.; Bietti, M.; Thiel, W.; Klussmann, M. Competitive Hydrogen Atom Transfer to Oxyl- and Peroxyl Radicals in the Cu-Catalyzed Oxidative Coupling of $\mathrm{N}$-Aryl Tetrahydroisoquinolines Using tertButyl Hydroperoxide. ACS Catal. 2016, 6, 3253.

11. Ratnikov, M. O.; Doyle, M. P. Mechanistic Investigation of Oxidative Mannich Reaction with tert-Butyl Hydroperoxide. The Role of Transition Metal Salt. J. Am. Chem. Soc. 2013, 135,1549 .

12. (a) Fosgerau, K.; Hoffmann, T. Peptide Therapeutics: Current Status and Future Directions. Drug. Discov. Today 2015, 20, 122. (b) Pollegioni, L.; Servi, S. Non-natural Amino Acids: Methods and Protocols; Springer: New York, 2012.

13. For selected reviews, see: (a) Guerrero, I.; Correa, A. Site-Selective Trifluoromethylation Reactions of Oligopeptides. Asian J. Org. Chem. 2020, 9, 898. (b) Gruß, H.; Sewald, N. LateStage Diversification of Tryptophan-Derived Biomolecules. 
Chem. Eur. J. 2020, 26, 5328. (c) Bottecchia, C.; Noël, T. Photocatalytic Modification of Amino Acids, Peptides, and Proteins. Chem. Eur. J. 2019, 25, 26. (d) Chow, H. Y.; Zhang, Y.; Matheson, E.; Li, X. Ligation Technologies for the Synthesis of Cyclic Peptides. Chem. Rev. 2019, 119, 9971. (e) Wang, W.; Lorion, M. M.; Shah, J.; Kapdi, A. R.; Ackermann, L. LateStage Peptide Diversification by Position-Selective C-H Activation. Angew. Chem. Int. Ed. 2018, 57, 14700. (f) Mondal, S.; Chowdhury, S. Recent Advances on Amino Acid Modifications via $\mathrm{C}-\mathrm{H}$ Functionalization and Decarboxylative Functionalization Strategies. Adv. Synth. Catal. 2018, 360, 1884. (g) deGruyter, J. N.; Malins, L. R.; Baran, P. S. Residue-Specific Peptide Modification: A Chemist's Guide. Biochemistry 2017, 56, 3863. (h) He, G.; Wang, B.; Nack, W. A.; Chen, G. Syntheses and Transformations of $\alpha$-Amino Acids via Palladium-Catalyzed Auxiliary-Directed $\mathrm{sp}^{3} \mathrm{C}-\mathrm{H}$ Functionalization. Acc. Chem. Res. 2016, 49, 635. (i) Noisier, A. F. M.; Brimble, M. A. C-H Functionalization in the Synthesis of Amino Acids and Peptides. Chem. Rev. 2014, 114, 8775.

14. (a) San Segundo, M.; Correa, A. Cross-Dehydrogenative Coupling Reactions for the Functionalization of $\alpha$-Amino Acid Derivatives and Peptides. Synthesis 2018, 50, 2853. (b) Brandhofer, T.; Mancheño, O. G. Site-Selective C-H Bond Activation/Functionalization of Alpha-Amino Acids and PeptideLike Derivatives. Eur. J. Org. Chem. 2018, 6050.

15. (a) Tan, M.; Li, K.; Yin, J.; You, J. Manganese/Cobalt-Catalyzed Oxidative $\mathrm{C}\left(\mathrm{sp}_{3}\right)-\mathrm{H} / \mathrm{C}\left(\mathrm{sp}^{3}\right)-\mathrm{H}$ Coupling: a Route to $\alpha-$ Tertiary $\beta$-Arylethylamines. Chem. Commun. 2018, 54, 1221. (b) Li, K.; Wu, Q.; Lan, J.; You, J. Coordinating Activation Strategy for $\mathrm{C}\left(\mathrm{sp}^{3}\right)-\mathrm{H} / \mathrm{C}\left(\mathrm{sp}^{3}\right)-\mathrm{H}$ Cross-Coupling to Access $\beta$ Aromatic $\alpha$-Amino Acids. Nat. Chem. 2015, 6, 8404. (c) Li, K.; Tan, G.; Huang, J.; Song, F.; You, J. Iron-Catalyzed Oxidative $\mathrm{C}-\mathrm{H} / \mathrm{C}-\mathrm{H}$ Cross-Coupling: An Efficient Route to $\alpha$-Quaternary $\alpha$-Amino Acid Derivatives. Angew. Chem. Int. Ed. 2013, 52, 12942. (d) Zhao, L.; Li, C.-J. Functionalizing Glycine Derivatives by Direct $\mathrm{C}-\mathrm{C}$ Bond Formation. Angew. Chem. Int. Ed. 2008, 47, 7075 .

16. (a) Guerrero, I.; Correa, A. Cu-Catalyzed Site-Selective $\mathrm{C}\left(\mathrm{sp}^{2}\right)-\mathrm{H}$ Radical Trifluoromethylation of Tryptophan-Containing Peptides. Org. Lett. 2020, 22, 1754. (b) San Segundo, M.; Correa, A. Pd-Catalyzed Site-Selective C $\left(\mathrm{sp}^{2}\right)-\mathrm{H}$ Radical Acylation of Phenylalanine Containing Peptides with Aldehydes. Chem. Sci. 2019, 10, 8872. (c) San Segundo, M.; Correa, A. Site-Selective $\mathrm{Cu}$-Catalyzed Alkylation of $\alpha$-Amino Acids and Peptides toward the Assembly of Quaternary Centers. ChemSusChem 2018, 11, 3893. (d) Guerrero, I.; San Segundo, M.; Correa, A. Iron-Catalyzed $\mathrm{C}\left(\mathrm{sp}^{3}\right)-\mathrm{H}$ Functionalization of $\mathrm{N}, \mathrm{N}$-Dimethylanilines with Isocyanides. Chem. Commun. 2018, 54, 1627. (e) Goitia, A.; Gómez-Bengoa, E.; Correa, A. Selective C $\left(\mathrm{sp}^{2}\right)-\mathrm{H}$ Halogenation of "Click" 4-Aryl-1,2,3-triazoles. Org. Lett. 2017, 19, 962.

17. San Segundo, M.; Guerrero, I.; Correa, A. Co-Catalyzed $\mathrm{C}\left(\mathrm{sp}^{3}\right)-\mathrm{H}$ Oxidative Coupling of Glycine and Peptide Derivatives. Org. Lett. 2017, 19, 5288.

18. For CDCs of $\alpha$-aminocarbonyl compounds with indoles, see for example: (a) Zhang, Y.; Ni, M.; Feng, B. Iron-Catalyzed Direct $\alpha$-Arylation of $\alpha$-Amino Carbonyl Compounds with Indoles. Org. Biomol. Chem. 2016, 14, 1550. (b) Huo, C.; Wang, C.; Wu, M.; Jia, X.; Xie, H.; Yuan, Y. Copper(I) Chloride-Catalyzed Aerobic Oxidative Arylation of Glycine Ester and Amide Derivatives. Adv. Synth. Catal. 2014, 356, 411. (c) Wang, Z.Q.; Hu, M.; Huang, X.-C.; Gong, L.-B.; Xie, Y.-X.; Li, J.-H. Direct $\alpha$-Arylation of $\alpha$-Amino Carbonyl Compounds with Indoles Using Visible Light Photoredox Catalysis. J. Org. Chem. 2012, 77, 8705. (d) Zhao, L.; Baslé, O.; Li, C.-J. Site-Specific
C-Functionalization of Free-(NH) Peptides and Glycine Derivatives via Direct $\mathrm{C}-\mathrm{H}$ Bond Functionalization. Proc. Natl. Acd. Sci. USA 2009, 106, 4106. For selected Co-catalyzed CDCs, see: (e) Li, Y.; Wang, M.; Fan, W.; Qian, F.; Li, G.; Lu, H. Cobalt-Catalyzed Cross-Dehydrogenative Coupling Reactions of (Benz)oxazoles with Ethers. J. Org. Chem. 2016, 81, 11743. (f) Kim, J. Y.; Cho, S. H.; Joseph, J.; Chang, S. Cobalt- and Manganese-Catalyzed Direct Amination of Azoles under Mild Reaction Conditions and the Mechanistic Details. Angew. Chem. Int. Ed. 2010, 49, 9899.

19. (a) Watanabe, E.; Kaiho, A.; Kusama, H.; Iwasawa, N. CobaltSalen Complex-Catalyzed Oxidative Generation of Alkyl Radicals from Aldehydes for the Preparation of Hydroperoxides. $J$. Am. Chem. Soc. 2013, 135, 11744. (b) Turrà, N.; Neuenschwander, U.; Baiker, A.; Peeters, J.; Hermans, I. Mechanism of the Catalytic Deperoxidation of tert-Butylhydroperoxide with Cobalt(II) Acetylacetonate. Chem. Eur. J. 2010, 16, 13226.

20. DFT studies have recently underpinned a resonance-based effect as the key factor of $N$-aryl groups in the success of oxidative Mannich-type reactions of tetrahydroisoquinolines: Tsang, A. S.-K.; Hashmi, A. S. K.; Comba, P.; Kerscher, M.; Chan, B.; Todd, M. H. N-Aryl Groups Are Ubiquitous in Cross-Dehydrogenative Couplings Because They Stabilize Reactive Intermediates. Chem. Eur. J. 2017, 23, 9313.

21. For the key role of computational studies in metal catalysis, see for example: (a) Vogiatzis, K. D.; Polynski, M. V.; Kirkland, J. K.; Townsend, J.; Hashemi, A.; Liu, C.; Pidko, E. A. Computational Approach to Molecular Catalysis by 3d Transition Metals: Challenges and Opportunities. Chem. Rev. 2019, 119, 2453. (b) Harvey, J. N.; Himo, F.; Maseras, F. Scope and Challenge of Computational Methods for Studying Mechanism and Reactivity in Homogeneous Catalysis, ACS Catal. 2019, 9 , 6803. (c) Sperger, T.; Sanhueza, I. A.; Schoenebeck, F. Computation and Experiment: A Powerful Combination to Understand and Predict Reactivities. Acc. Chem. Res. 2016, 49, 1311.

22. See, for example: (a) Houghton, R. P.; Rice, C. R. Cobalt(II)Catalysed Decomposition of Hydroperoxides. Implications for Alkane Functionalization. Polyhedron 1996, 15, 1893. (b) Minisci, F.; Fontana, F.; Araneo, S.; Recupero, F.; Banfi, S.; Quici, S. Kharasch and Metalloporphyrin Catalysis in the Functionalization of Alkanes, Alkenes, and Alkylbenzenes by t$\mathrm{BuOOH}$. Free Radical Mechanisms, Solvent Effect, and Relationship with the Gif Reaction. J. Am. Chem. Soc. 1995, 117, 226. (c) Scott, E. J. Y. Kinetics of the reactions of cobalt(II) and cobalt(III) acetates with benzyl hydroperoxide in acetic acid at 25.deg.. J. Phys. Chem. 1970, 74, 1174. (d) Kharasch, M. S.; Fono, A. Metal Salt-Induced Homolytic Reactions. I. A New Method of Introducing Peroxy Groups into Organic Molecules. J. Org. Chem. 1959, 24, 72.

23. (a) Avila, D. V.; Ingold, K. U.; Lusztyk, J.; Green, W. H.; Procopio, D. R. Dramatic Solvent Effects on the Absolute Rate Constants for Abstraction of the Hydroxylic Hydrogen Atom from tert-Butyl Hydroperoxide and Phenol by the Cumyloxyl Radical. The Role of Hydrogen Bonding. J. Am. Chem. Soc. 1995, 117, 2929. (b) Paul, H.; Small Jr., R. D.; Scaiano, J. C. Hydrogen abstraction by tert-butoxy radicals. A Laser Photolysis and Electron Spin Resonance Study. J. Am. Chem. Soc. 1978, 100, 4520 .

24. (a) Liu, X.-X.; Wu, Z.-Y.; He, Y.-Q.; Zhou, X.-Q.; Hu, T.; Ma, C.-W.; Huang, G.-S. Copper-Catalyzed C-N Bond Formation via Oxidative Cross-Coupling of Amines with $\alpha$-Aminocarbonyl Compounds. Adv. Synth. Catal. 2016, 358, 2385. (b) Wei, W.-T.; Song, R.-J.; Li, J.-H. Copper-Catalyzed Oxidative $\alpha$-Alkylation of $\alpha$-Amino Carbonyl Compounds with Ethers via 
Dual C $\left(\mathrm{sp}^{3}\right)-\mathrm{H}$ Oxidative Cross-Coupling. Adv. Synth. Catal. 2014, 356, 1703.

25. For further details, see the Supporting Information.

26. Mujika, J.-I.; Uranga, J.; Matxain, J.-M.; Computational Study on the Attack of $\mathrm{OH}$ Radicals on Aromatic Amino Acids. Chem. Eur. J. 2013, 19, 6862.

27. According to our calculations, the HAT-1 step with $t \mathrm{BuOO}^{\circ}$ is around $20 \mathrm{kcal} / \mathrm{mol}$ unfavorable with respect to the parent process with $t \mathrm{BuO}^{\circ}$, hence the first hydrogen abstraction step is more likely to occur with $t \mathrm{BuO}^{\circ}$ species.

28. Although experimental evidences seemed to rule out the feasibility of the alternative route $a$, we did perform some calculations to have a more fundamental rationale. In this respect, the reaction enthalpy for the direct radical coupling of Int-H with $t \mathrm{BuOO}^{\circ}$ species was found to be $-43.92 \mathrm{kcal} / \mathrm{mol}$. However, according to the energetics shown in Figure 1, the in-situ formed $\mathrm{Co}(\mathrm{III})-\mathrm{OH}$ complex is more likely to undergo a reduction reaction with Int-H than a SET-2, which could prevent the formation of $t \mathrm{BuOO}^{\circ}$ and hence favor the formation of the imine intermediate in the reaction medium.

29. Spier, E.; Neuenschwander, U.; Hermans, I. Insights into the Cobalt(II)-Catalyzed Decomposition of Peroxide. Angew. Chem. Int. Ed. 2013, 52, 1581. 